\title{
PICOSECOND KINETICS OF STATE-SELECTIVE SINGLET OXYGEN PRODUCING PHOTOCHEMISTRY OF AROMATIC ENDOPEROXIDES
}

\author{
E.V. SITZMANN, J.G. LANGAN ', D.A. HROVAT ${ }^{2}$ and K.B. EISENTHAL \\ Department of Chemistry, Columbia University, New York, NY 10027, USA
}

Received 7 June 1989; in final form 24 July 1989

\begin{abstract}
Picosecond measurements show that the photo-fragmentation of anthracene endoperoxides leading to the anthracene moiety and singlet oxygen does not occur in a single step, but involves a short-lived intermediate. The upper excited state dual bond dissociation kinetics of the endoperoxides of 9,10-diphenylanthracene $\left(9,10-\mathrm{DPA}-\mathrm{O}_{2}\right)$ and of 9-phenylanthracene $\left(9,10-\mathrm{PA}-\mathrm{O}_{2}\right)$

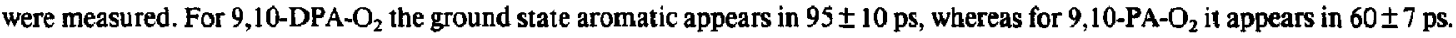
The results are consistent with our earlier proposed mechanism for the dissociation from the upper excited singlet states of isomers of the anthracene endoperoxides.
\end{abstract}

\section{Introduction}

The observation of electronic state selective chemistry provides valuable insight into the nature of chemical reactions. For cases in which the photochemistry depends on the wavelength of the irradiating light the initially excited state not only determines the pathway of a chemical reaction but also requires the existence of an extremely efficient chemical reaction to compete with rapid energy relaxation processes.

Aromatic endoperoxides are compounds known to undergo wavelength-dependent photochemistry [1-11]. Steady state quantum yield measurements of the products showed that exciting into $S_{1}$ gives products derived from $\mathrm{O}-\mathrm{O}$ rupture, but excitation to higher states gives ${ }^{1} \mathrm{O}_{2}$ and the anthracene moiety, as shown in scheme 1 . We have extended our studies of these molecules, characterized by an $\mathrm{O}-\mathrm{O}$ peroxide bridge, to better understand the nature of the photofragmentation process and its dependence on the wavelength of the exciting light. We were also motivated by the importance of these compounds as

1 Present address: Air Products \& Chemicals, Allentown, PA 18195, USA.

2 Present address: Department of Chemistry, University of Washington, Seattle, WA 98105 , USA. a useful source of singlet molecular oxygen, ${ }^{1} \mathrm{O}_{2}$, an active species in the degradation of chemical and biological substances and as an energy pump for the high-powered iodine laser [12].

A crucial issue in describing the observed chemistry following photoexcitation is to know the chemical species first produced. Does the photodissociation lead directly to the expected singlet oxygen intermediate, or is it preceded by other intermediates which could themselves undergo reactions other than the one leading to the anticipated intermediate? It was widely assumed that photodissociation of endoperoxides involved the simultaneous rupture of both $\mathrm{C}-\mathrm{O}$ bonds leading directly to ${ }^{\prime} \mathrm{O}_{2}$ and the ground state aromatic fragment [13-15]. We have found $[11,16]$ that this is not the case for 1,4dimethyl-9,10-diphenylanthracene endoperoxides (DMDPA- $\mathrm{O}_{2}$ ) but that there is an earlier step in the process, as shown in scheme 2 . Important new experimental evidence for the two-step mechanism was also recently found for the upper excited state de-

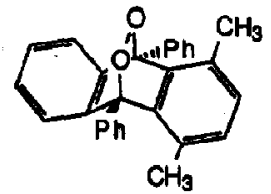

9,10-DMDPA-O

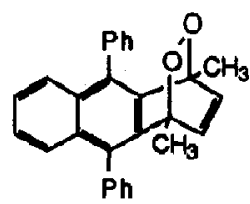

1,4-DMDPA-O $\mathrm{O}_{2}$ 

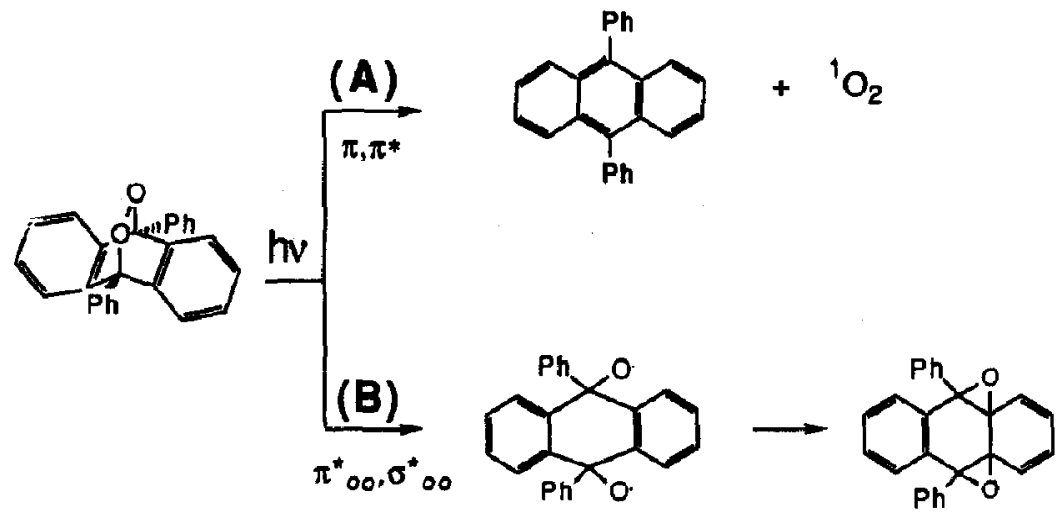

Scheme 1. State selective photodecomposition pathways for aromatic endoperoxides: $(A) S_{n}(n \geqslant 2)$ excited state decay to produce ${ }^{1} \mathrm{O}_{2}$ and the aromatic moiety, and (B) $S_{1}$ excited state decay via $\mathrm{O}-\mathrm{O}$ bond rupture.

$\mathrm{AO}_{2} \stackrel{h \nu}{\rightarrow}\left(\mathrm{AO}_{2}\right)^{*} \stackrel{5 \mathrm{ps}}{\longrightarrow}[$ intermediate $] \stackrel{50-100 \mathrm{ps}}{\longrightarrow} \mathrm{O}_{2}+\mathrm{A}$

Scheme 2. Upper excited state $\left(S_{n}, n \geqslant 2\right)$ photodecomposition of anthracene endoperoxides $\left(\mathrm{AO}_{2}\right)$.

composition of other aromatic endoperoxides as well $[17,18]$.

To extend the generality of our results on the anthracene endoperoxide isomers we measured the formation kinetics of the aromatic moiety for phenyl substituted derivatives of the 9,10-anthracene endoperoxide. In particular we examined the 9,10-endoperoxide but without the methyl groups in the 1,4 positions, i.e. 9,10-DPA-O $\mathrm{O}_{2}$. The known wavelength dependence of this anthracene derivative is similar to the isomer 9,10-DMDPA-O ${ }_{2}$, which we previously studied. We wish to establish whether the kinetics are the same and if so thereby establish a relationship between the observed electronic spectra and the kinetics of dissociation. Furthermore in order to better understand the nature of the photofragmentation process, its relation to the structure of the endoperoxide, and the dependence of the photodissociation pathways on the excitation wavelength we examined the 9-phenylanthracene derivative $\left(9,10-\mathrm{PA}-\mathrm{O}_{2}\right)$.

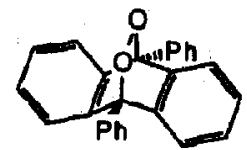

9,10-DPA-O 2

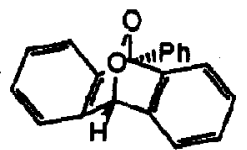

9,10-PA-O2

\section{Results and discussion}

Direct observation of the kinetics of anthracene formation following UV laser photodecomposition of endoperoxides 9,10-DPA-O $\mathrm{O}_{2}$ and 9,10-PA-O $\mathrm{O}_{2}$ was accomplished using the laser-induced fluorescence (LIF) method [11]. The experiment consisted of irradiation of a flowed, deoxygenated acetonitrile solution of the endoperoxide $\left((0.8-1) \times 10^{-3} \mathrm{M}\right)$ with a picosecond laser pulse at $266 \mathrm{~nm}$ from a passively mode-locked Nd:YAG laser (25-30 ps fwhm, <0.01 $\mathrm{mJ})$. The formation rate of the anthracene derivative was monitored by laser-induced fluorescence using a weak probe pulse at $355 \mathrm{~nm}$ at variable time delays. The results of the picosecond dynamic measurements are shown in fig. 1 .

Using the measured system response a best fit to the LIF data was obtained for an exponential risetime of $95 \pm 10 \mathrm{ps}$ for $9,10-\mathrm{DPA}-\mathrm{O}_{2}$ and $60 \pm 7 \mathrm{ps}$ for 9,10-PA- $\mathrm{O}_{2}$. These experiments demonstrate that the formation of the parent hydrocarbon proceeds at a non-instantaneous rate following photoexcitation.

Of special importance in endoperoxide photochemistry is the dependence of the product yields on excitation wavelength. Previous investigations [2,3] found for 9,10-DPA- $\mathrm{O}_{2}$ that the fraction of anthracene in the photoproducts depended on the irradiating wavelength. Excitation into the $S_{0} \rightarrow S_{1}$ $\left(\pi_{O O}^{*}, \sigma_{00}^{*}\right)$ band results in predominant formation of the products derived from the cleavage of the $\mathrm{O}-$ $O$ bond (diepoxides, and secondary rearrangements, 


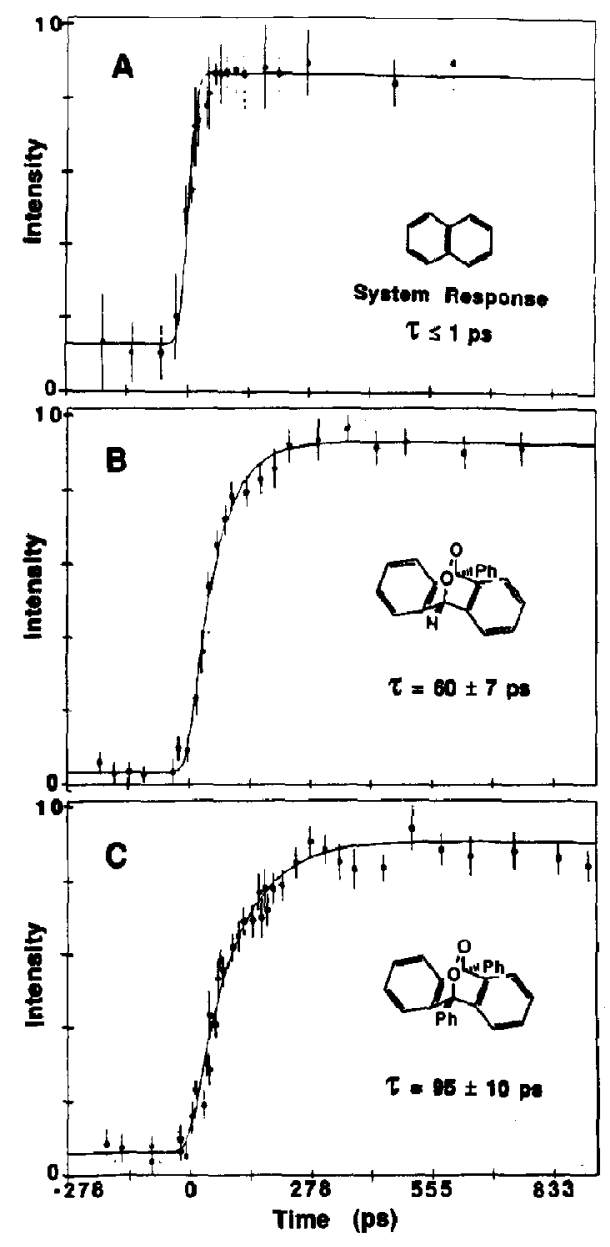

Fig. 1. (A) System response, obtained from $S_{1} \rightarrow S_{n}$ absorption of naphthalene; (B) formation kinetics of 9-phenylanthracene following picosecond laser irradiation at $266 \mathrm{~nm}$ of $9,10-\mathrm{PA}_{-} \mathrm{O}_{2}$ in acetonitrile at ambient temperature: (C) formation kinetics of 9,10-diphenylanthracene following picosecond laser irradiation at $266 \mathrm{~nm}$ of $9,10-\mathrm{DPA}-\mathrm{O}_{2}$ in acetonitrile at ambient temperature.

etc.). The absorption at wavelengths less than 294 $\mathrm{nm}\left(\epsilon=10^{3}-10^{4} \mathrm{M}^{-1} \mathrm{~cm}^{-1}\right)$ corresponds to the $S_{0} \rightarrow S_{2}\left(\pi, \pi^{*}\right)$ transition. Irradiation into this band resulted in predominant formation of 9,10-DPA and ${ }^{1} \mathrm{O}_{2}$. The yield of these two products was constant down to $254 \mathrm{~nm}$. These results indicate that photofragmentation of $9,10-\mathrm{DPA}-\mathrm{O}_{2}$, to produce 9,10 DPA and $\mathrm{O}_{2}$, is a state selective process occurring from an upper excited singlet state of the endoperoxide $\left(S_{n}, n \geqslant 2\right)$. Since the upper excited $S_{n}$ state is short lived, the chemical deactivation pathway must be extremely efficient in order to compete with rapid radiationless relaxation to the lower energy states of the endoperoxide [19]. Indeed, the quantum yield to produce oxygen and the anthracene has a value of 0.28 [2] when the endoperoxide is directly excited to the upper $\pi, \pi^{*}\left(S_{n}, n \geqslant 2\right)$ state, further attesting to the efficiency of the reaction. From our time-resolved measurements we know that the formation rate of the anthracene takes $95 \pm 10$ ps after direct excitation into the short-lived $\pi, \pi^{*}$ state of the endoperoxide. In light of these facts we are forced to conclude that simultaneous formation of singlet oxygen and the ground state anthracene does not occur instantaneously, and thus does not follow a concerted mechanism as theoretically described by Kearns and Kahn [13-15].

We are left to consider two possible reaction pathways (scheme 3 ) that would be consistent with the observed kinetics of the delayed formation of the anthracene moiety. In one case there is a sequential $\mathrm{C}$ $O$ bond cleavage, which involves a 1,6-biradical intermediate. The two components of the 1,6-biradical intermediate are describable by a linked aromatic carbon radical and a peroxy radical. The decay of this biradical would be by recombination and/or by breaking the second $\mathrm{C}-\mathrm{O}$ bond to produce ${ }^{1} \mathrm{O}_{2}$ and the ground state aromatic hydrocarbon.

The second case involves rapid expulsion of ${ }^{1} \mathrm{O}_{2}$ from the excited state endoperoxide leaving an excited valence anthracene isomer (which we describe as having a 1,4-aromatic biradicaloid structure). The excited valence isomer lifetime would then account for the slow formation time of the ground state anthracene. We differentiate this pathway from a concerted one of Kearns and Kahn [13-15] because the ground state anthracene is not produced simultaneously with ${ }^{1} \mathrm{O}_{2}$. We further point out that we also rule out a simple hemi-Dewar form [20-27] for the valence anthracene isomer, since the lifetimes (50$100 \mathrm{ps}$ ) that we observe are much shorter than the re-aromatization times found for ground state Dewar isomers, which are typically on the order of $10^{-6}-10^{-3}$ s $[20-27]$.

The difference in the kinetics of 9,10-DPA- $\mathrm{O}_{2}$ and 9,10-PA- $\mathrm{O}_{2}$ is of particular interest. We find that the aromatic moiety is formed faster for $9,10-\mathrm{PA}-\mathrm{O}_{2}$ compared to the diphenyl-substituted $9,10-\mathrm{DPA}_{2} \mathrm{O}_{2}$ 


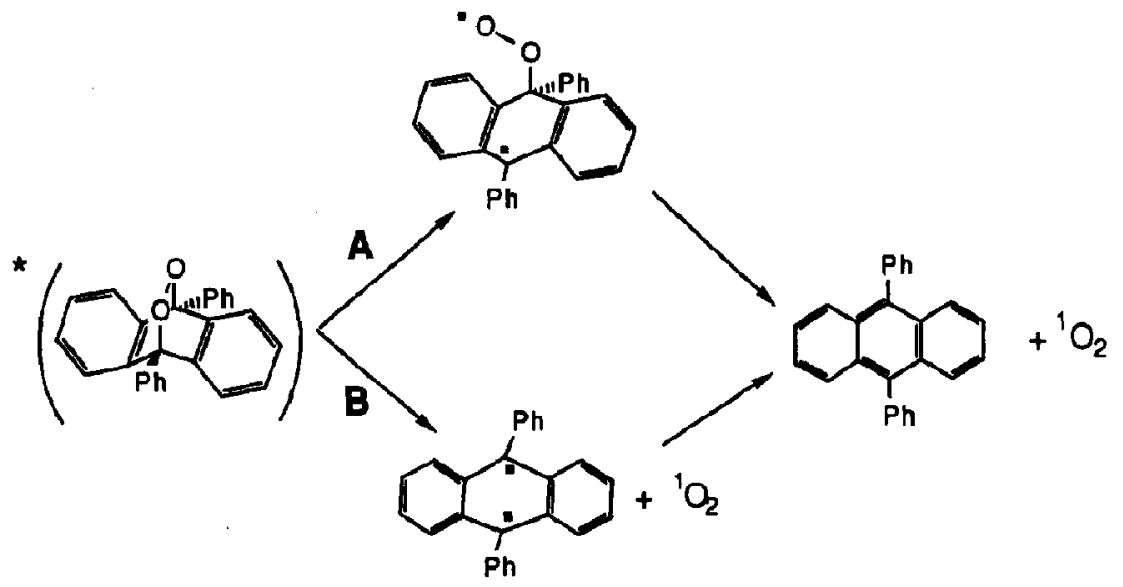

Scheme 3. Biradical mechanism for upper excited state $\pi, \pi^{*}$ state photofragmentation of aromatic endoperoxides. (A) Sequential $C-O$ bond rupture to produce 1,6-biradical, or (B) fast rupture of both $\mathrm{C}-\mathrm{O}$ bonds to produce ${ }^{\prime} \mathrm{O}_{2}$ and an excited anthracene valence isomer.

derivative. Such differences in the kinetics, due to the change in structure, was previously observed for the case of the isomers of the DMDPA- $\mathrm{O}_{2}$ endoperoxide. The different kinetics in the derivatives may be resulting from changes in the stability of the shortlived intermediate produced in the reaction sequence. Evidence for this is based on the fact that the lifetime of the precursor $\pi, \pi^{*}$ state is $<5 \mathrm{ps}$, and thus the rate determining step in the production of the aromatic moiety will be determined by the lifetime of the intermediate (scheme 2). At issue now is to determine whether there is a correlation between the molecular structure and the observed intermediate lifetimes.

If a biradical intermediate (either as the 1,6-peroxy-aromatic or as the 1,4-aromatic type) is involved in the pathway leading from $S_{2}$ to the final photoproducts, as proposed by us $[11,16]$ and others $[17,18]$, we can explain the observed kinetics in terms of how the molecular structure affects the biradical stability and thereby affect its lifetime. For instance, within the biradical model the lifetime of the intermediate decreases when there is either an increase in steric repulsions and/or decrease in resonance stabilization [28-30]. From table 1 we find that the longest lived intermediate is observed for the 9,10-DPA- $\mathrm{O}_{2}$ derivative ( $95 \pm 10 \mathrm{ps).} \mathrm{We} \mathrm{inter-}$ prete this long lifetime as being a result of the stabilizing influence due to resonance interactions associated with the triphenyl methyl radical like center that would be produced following $\mathrm{C}-\mathrm{O}$ bond cleavage (see table 2). A similar resonance effect should also be present for the 9,10-DMDPA-O $\mathrm{O}_{2}$ as well. However, a shorter intermediate lifetime of $75 \pm 20$ ps is found for the 9,10-DMDPA- $\mathrm{O}_{2}$ derivative, which can be explained as resulting from the destabilizing influence of steric interactions between the methyl and phenyl groups in the biradical (see table 2). Thus, although the biradical intermediate produced from 9,10-DMDPA-O $\mathrm{O}_{2}$ should be stabilized by the strong resonance stabilization due to the triphenyl methyl like radical centers (as it would be for $9,10-\mathrm{DPA}-\mathrm{O}_{2}$ ), the destabilizing influence due to steric interactions present means that it will be shorter lived.

The effect of resonance interactions (or lack thereof ) also explains the ordering of lifetimes observed for the other derivatives, as listed in table 1.

Table I

Formation time of the ground state anthracene moiety following $S_{0} \rightarrow S_{n}(n \geqslant 2)$ excitation, at $266 \mathrm{~nm}$, of various endoperoxides in acetonitrile at ambient temperature

\begin{tabular}{ll}
\hline Endoperoxide $^{\text {a) }}$ & Risetime (ps) \\
\hline $9,10-\mathrm{DPA}^{-\mathrm{O}_{2}}$ & $95 \pm 10^{\mathrm{b}}$ \\
$9,10-\mathrm{DMDPA}^{\mathrm{O}} \mathrm{O}_{2}$ & $75 \pm 20^{\mathrm{c}}$ \\
$9,10-{\mathrm{PA}-\mathrm{O}_{2}}$ & $60 \pm 7^{\mathrm{b}}$ \\
$1,4-\mathrm{DMDPA}-\mathrm{O}_{2}$ & $50 \pm 15^{\mathrm{c}}$ \\
\hline
\end{tabular}

a) See text for structures. "This work. c) ref. [11]. 
Table 2

Intermediate carbon radical centers derived from anthracene endoperoxide derivatives

Endoperoxide precursor

For instance, the $60 \pm 7 \mathrm{ps}$ lifetime observed for the 9,10-PA- $\mathrm{O}_{2}$ derivative is shorter than observed for the 9,10-diphenyl anthracene derivatives, i.e. 9,10DPA-O ${ }_{2}\left(95 \pm 10\right.$ ps ) or 9,10-DMDPA-O ( $_{2}(75 \pm 20$ ps). This result is consistent with the idea that for 9,10-PA- $\mathrm{O}_{2}$ we would produce a diphenyl methyl like radical upon $\mathrm{C}-\mathrm{O}$ cleavage at the $\mathrm{C}-10$ position, whose stability is less than the triphenyl methyl radical like centers for the 9,10-diphenyl anthracene endoperoxide precursors.

Finally, when 1,4-DMDPA- $\mathrm{O}_{2}$ undergoes $\mathrm{C}-\mathrm{O}$ bond cleavage it will produce a biradical that lacks the enhanced resonance stabilization effects expected for the biradicals derived from either 9,10DPA-O $, 9,10-D M D P A-\mathrm{O}_{2}$ or $9,10-\mathrm{PA}_{2} \mathrm{O}_{2}$, i.e. it lacks the multiple phenyl groups being bonded directly to the carbon radical center. The short $50 \pm 15$ ps risetime observed for the formation time of the aromatic moiety in 1,4-DMDPA- $\mathrm{O}_{2}$ is consistent with this explanation.

\section{Conclusions}

Picosecond measurements show that excited state fragmentation of anthracene endoperoxides leading to the aromatic moiety and singlet oxygen is a multistep process involving a short-lived intermediate. The observation of the delayed appearance of the anthracenc following photoexcitation into the upper $\pi, \pi^{*} \mathrm{~S}_{n}(n \geqslant 2)$ states as well as structural effects on the kinetics can be explained by an intermediate that is formed either by non-concerted, sequential $\mathrm{C}-\mathrm{O}$ bond rupture, which involves a 1,6-biradical intermediate, or by the formation of an excited state anthracene valence isomer that has substantial biradical character.

\section{Acknowledgement}

We gratefully acknowledge the National Science Foundation, the Air Force Office of Scientific Research and the donors of the Petroleum Research Fund, administered by the American Chemical Society, for their generous support of this work.

\section{References}

[1] J. Rigaudy, C. Brelière and P. Schribe, Tetrahdron Letters (1978) 687

[2] W. Drews, R. Schmidt and H.-D. Brauer, Chem. Phys. Letters 70 (1980) 84.

[3] J. Rigaudy, P. Scribe and C. Brelière, Tetrahdron 37 (1981) 2585.

[4] R. Schmidt, W. Drews and H.-D. Brauer, J. Photochem. 18 (1982) 365 .

[5] R. Schmidt, J. Photochem. 23 (1983) 379.

[6] H.-D. Brauer and R. Schmidt, J. Photochem. 27 (1984) 17.

[7] R. Gabriel, R. Schmidt and H.-D. Brauer, Z. Physik. Chem. NF 141 (1984) 41.

[8] R. Schmidt, H.-D. Brauer and J. Rigaudy, J. Photochem. 34 (1986) 197.

[9] R. Schmidt, K. Schaffner, W. Trost and H.-D. Brauer, J. Phys. Chem. 88 (1984) 956.

[10] J. Rigaudy, A. Casper, J. Baranne-Lafont and C. Chassagnard, Bull. Soc. Chim. France 2 (1984) 195.

[11] K.B. Eisenthal, N.J. Turro, C.G. Dupuy, D.A. Hrovat, J. Langan, T.A. Jenny and E.V. Sitzmann, J. Phys. Chem. 90 (1986) 5168

[12] H.H. Wasserman and R.W. Murray, Singlet oxygen (Academic Press, New York, 1979).

[13] D.R. Kearns and A.U. Kahn, Photochem. Photobiol. 10 (1969) 193.

[14] D.R. Kearns, Chem. Rev. 7 ( (1971) 395.

[15] D.R. Kearns, J. Am. Chem. Soc. 91 (1969) 6554. 
[16] E.V. Sitzmann, C. Dupuy, Y. Wang and K.B. Eisenthal, in: Picosecond phenomena, Vol. 3, eds. K.B. Eisenthal, R.M. Hochstrasser, W. Kaiser and J. Laberau (Springer, Berlin, $1982)$ p. 168.

[ 17] Th. Blumenstock, F.J. Comes, R. Schmidt and H.-D. Brauer, Chem. Phys. Letters 127 (1986) 452.

[18] Th. Blumenstock, K. Jesse, F.J. Comes, R. Schmidt and H.* D. Brauer, Chem. Phys. 130 (1989) 289.

[19] M. Kasha, Discussions Faraday Soc. 9 (1950) 14.

[20] S.L. Susan and J. Michl, in: Photochemistry and Photobiology, Proceedings of the International Conference, Vol. 2, ed. A.H. Zewail (Harwood, Chur, 1983) p. 1191.

[21 ] J.E. Anderson and R.W. Franck, Nouv. J. Chim. 3 (1979) 717.

[22] M. Meisl and R. Janoschek, J. Chem. Soc. Chem. Commun. (1986) 1066 .
[23] H. Dreeskamp, B. Jahn and J. Pabst, Z. Naturforsch. 36 A (1981) 665 .

[24] B. Jahn and H. Dreeskamp, Ber. Bunsenges. Physik. Chem. 88 (1984) 42.

[25] W. Pritschins and W. Grimme, Tetrahedron Letters 23 (1982) 1151 .

[26] H. Güsten, M. Mintas and L. Klasinc, J. Am. Chem. Soc. 102 (1980) 7936.

[27] B. Sztuba and E. Ratajczak, J. Chem. Soc. Perkin Trans. II (1982) 823 .

[28] D. Griller and K.U. Ingold, Accounts Chem. Res. 9 (1976) 13.

[29] H.G. Viehe, Z. Janousek and R. Merenyi, eds., Substituent effects in radical chemistry, NATO ASI Series, Series C, Vol. 189 (Reidel, Dordrecht, 1986).

[30] V. Balasubramaniyan, Chem. Rev. 66 (1966) 567. 